\title{
Educación social y escuela en España. A propósito de la formación e inserción laboral
}

\author{
María D. Dapía Conde; María R. Fernández González *
}

Resumen. La Educación Social es una profesión reciente en el estado español que está en construcción. A sus tres ámbitos de intervención tradicionales -Educación de Personas Adultas, Animación Sociocultural y Pedagogía del Ocio, y Educación Especializada-, se le han sumado otros ámbitos emergentes, como la escuela. En España, identificamos tres modelos diferentes seguidos en la incorporación de los/as educadores/as sociales en los centros escolares: a) educadores/as sociales adscritos de forma institucional, formando parte de los recursos humanos propios; b) educadores/as sociales pertenecientes a los Servicios Sociales municipales; y, c) proyectos liderados por educadores/as sociales, que se llevan a cabo en los centros escolares mediante entidades intermediarias, previa firma de un convenio con la administración autonómica educativa. Siendo una realidad la incorporación de la Educación Social a la escuela; aunque de forma dispar según las comunidades autónomas, nos plateamos, si la formación inicial que reciben los/as educadores/as sociales responde a las exigencias planteadas en la escuela en comunidades con tradiciones diferentes. Tras analizar los planes de estudio, los resultados indican que no se constatan diferencias en la formación y además esta formación responde a las exigencias de las convocatorias (temarios) así como a las funciones, con alguna adaptación necesaria. En consecuencia, la incorporación de la educación social en el ámbito escolar, fundamentalmente en educación secundaria existe, aunque permanece la necesidad de definir más precisamente el desempeño profesional (funciones), ampliar la plantilla haciéndola extensiva a más centros y continuar la reflexión acerca de una formación adecuada a las nuevas exigencias profesionales.

Palabras clave: formación inicial; escuela; universidad; educación social; profesionalización.

EDUCAÇÃO SOCIAL E ESCOLA NA ESPANHA. NO TOCANTE A FORMAÇÃO E INSERÇÃO NO MERCADO DE TRABALHO

Resumo. A Educação Social é uma profissão recente no estado espanhol que está em construção. Os seus três campos tradicionais de intervenção - Educação de Adultos, Animação Sociocultural e Pedagogia do Lazer, e Educação Especializada - contaram com a adesão de outros campos emergentes tal como a escola. Na Espanha, identificamos três modelos diferentes seguidos pela incorporação dos(as) educadores(as) sociais nas escolas: a) educadores(as) sociais integrados de forma institucional, que fazem parte dos recursos humanos próprios; b) educadores(as) sociais

\footnotetext{
* Universidad de Vigo, España
} 
pertencentes aos Serviços Sociais municipais; e c) projetos liderados por educadores(as) sociais, que são realizados nas escolas por meio de entidades intermediárias, após a assinatura de um convênio com o órgão público estadual de educação. Sendo uma realidade a incorporação da Educação Social à escola, embora de forma diferente de acordo com as comunidades autônomas, verificamos se a formação inicial dos(as) educadores(as) sociais atende às exigências da escola em comunidades que tenham costumes diferentes. Após analisar os planos de estudo, os resultados indicam que não existem diferenças de formação e que, esta formação também atende às exigências dos editais (programas), bem como às funções, com alguma adaptação necessária. Consequentemente, existe a incorporação da educação social no meio escolar, principalmente no ensino secundário, embora permaneça a necessidade de definir mais precisamente o desempenho profissional (funções), aumentar o número de funcionários em mais escolas e continuar a refletir sobre uma formação adequada às novas exigências do mercado de trabalho

Palavras-chave: formação inicial; escola; universidade; educação social; profissionalização.

\section{SOCIAL EDUCATION AND SCHOOL IN SPAIN. CONCERNING TRAINING AND LABOR INSERTION}

Abstract. Social Education is a recent profession in the Spanish State which is under construction. To its three traditional areas of intervention-Education of Adult People, Sociocultural Animation and Pedagogy of the Leisure, and Specialized Education-, other emerging areas have been added, like the school. In Spain, we identified three different models following each other's in the incorporation of social educators in the schoolchildren centers: a) social educators assigned institutionally, being part of our own human resources; b) social educators belonging to the Municipal Social Services; and, c) projects lead by social educators, which are carried on the centers schools through intermediary entities, after signing an agreement with the educational autonomic administration. Being a reality the incorporation from Social Education to the school; although in a dissimilar way according to the Autonomous Communities, we planted, if the initial training which is received by social educators responds to the demands raised into the school in communities with different traditions. After analyzing the study programs, the results indicate that there are no differences in the training and in addition this training responds to the requirements of calls (syllabus) as well as roles, with some required adjustment. Consequently, the inclusion of social education exists in the school milieu, mainly in secondary school, although there remains the need to define more precisely professional performance (roles), expand the workforce being applicable to more centers and continue the reflection about an appropriate training to the new professional demands.

Keywords: Initial training; school; university; social education; professionalization 


\section{NUEVOS PERFILES EN EDUCACIÓN SOCIAL}

La educación social es una profesión educativa reciente y emergente en el estado español que se sigue desarrollando, está en construcción. Aunque ya existían tradiciones vinculadas a lo que hoy identificamos como educación social, no es hasta el año 1991, con la aprobación del título universitario de diplomado en educación social, mediante RD 1420/1991, cuando oficialmente aparece en España la educación social. En él se recoge que "las enseñanzas conducentes a la obtención del título oficial de diplomado en educación social deberán orientarse a la formación de un educador en los campos de la educación no formal, educación de adultos (incluidos los de la tercera edad), inserción social de personas desadaptadas y minusválidos, así como en la acción socioeducativa" (directriz primera). La reflexión inicial que se desprende de esta regulación es la de ser considerada un avance en la formación y dignificación de la educación social, pero a la vez, es criticada por su carácter confuso, vago e impreciso en la definición del perfil profesional y, esta indefinición es particularmente preocupante al referirnos al ámbito escolar.

Según el RD citado, la educación social se asocia a la educación no formal, obviando, por tanto, el sistema escolar, y pudiendo únicamente vincularse por la «acción socioeducativa». Esta ruptura entre escuela (educación formal) y educación no formal forma parte de nuestra historia pedagógica, tal como recoge Merino (2013), cuando afirma que "en la tradición conceptual de la pedagogía había una distinción clara entre educación formal y educación no formal" (p. 2), la escuela pertenecía a la primera y la educación social se centraba en la segunda. Esta distinción presupone una conceptualización esencialista o estática, que no tiene en cuenta el contexto sociohistórico y lo dinámico del hecho educativo, siendo claramente debatida.

Casi desde la aprobación del título de diplomado en educación social se cuestiona el reduccionismo de identificar a la educación social con la educación no formal (Trilla, 1996; Petrus, 2000), aumentado las voces más recientemente, tanto desde la universidad como desde el colectivo profesional, al entender que no solo es posible una pedagogía social en el ámbito escolar, sino que es su lugar natural (March y Orte, 2014). Los argumentos esgrimidos en este sentido podrían resumirse del modo siguiente: desde la academia, en la idea expuesta por Ortega (2005), defendiendo que, en general, toda educación es o debe ser social, ya que, supone una progresiva y continua configuración de la persona para ser y convivir con los demás que acontece y se desarrolla a lo largo de la vida, por lo que "no se da, pues, única y exclusivamente en una determinada etapa de la vida, ni se circunscribe sólo a la escuela. Y es en el continuum de la «educación a lo largo de la vida» donde se inserta la educación social" (p. 114); y, desde 
los y las profesionales de la Educación Social, en la conceptualización aportada por la Asociación Estatal de Educación Social (ASEDES, 2007), que definen la Educación Social como un derecho de la ciudadanía, generadora de contextos educativos y acciones mediadoras y formativas, que posibilitan el desarrollo de la sociabilidad y la circulación social, así como la promoción cultural y social para ampliar las perspectivas educativas, laborales, de ocio y participación social.

Ante esta nueva realidad, las definiciones más recientes de Educación Social reflejan su dinamismo y su capacidad para dar respuesta a las nuevas necesidades sociales. Según Molina y Blázquez (2015), la educación social "atiende a la producción de efectos de inclusión cultural, social y económica, al dotar a las personas de los recursos pertinentes para resolver los desafíos y retos del momento histórico y el marco social en el que viven" (p. 44), y definen a los educadores y educadoras sociales como unos profesionales que median entre las exigencias del espacio social y las personas que habitan en él, al propiciar el conocimiento de los saberes y herramientas que toda persona necesita para vivir en esta sociedad global y local. Por lo que, no sólo deben conocer los recursos y servicios que lo social ofrece a su ciudadanía, sino que deben "saber acompañar, orientar y, sobre todo, enseñar a las personas a utilizar, disfrutar y hacer suyos esos bienes culturales y los recursos que el marco social les posibilita" (p. 44).

La complejidad de la definición de la profesión de educación social según el Real Decreto 1420/1991, junto a las ansias por delimitar su espacio profesional, impulsan el reconocimiento y consolidación de tres ámbitos específicos de intervención de la Educación Social: Educación de Personas Adultas, Animación Sociocultural y Pedagogía del Ocio, y Educación Especializada. Estos ámbitos clásicos se han visto complementados con otros ámbitos emergentes, por ejemplo, deportes como instrumento de socialización, inteligencia emocional, educación ambiental, educación intercultural, mediación, acogida y adopción, mujeres (promoción y maltrato), municipio y movimiento asociativo, TICs y medios audiovisuales de comunicación con repercusiones en procesos de socialización o inclusión de la Educación Social dentro del marco escolar y de los Servicios Sociales (Amador et al., 2014; Chozas, 2003, Esteban y Amador, 2017, Froufe, 1997; Gómez, 2003; Longás, 2000; March y Orte, 2002; Pérez, 2003; Petrus, 2000; Senent, 2003; Parcerisa, 2008).

Centrándonos en la intervención de la educación social en la escuela, ésta se ve refrendada, por una parte, por los propios fines de la educación, señalados en el artículo 2 de la ley de educación vigente en España (LOMCE, 
2013), ya que, según Ortega (2014) y López (2013), las funciones y fines de la escolarización coinciden en general con los fines generales atribuibles a la Educación Social.

Por otra parte, no sólo la contribución que puede hacer la Educación Social al cumplimiento de estos fines justifica la necesidad de incluir a los educadores y educadoras sociales como profesionales de los centros escolares, sino que la realidad de la escuela como institución social y las cada vez más desmesuradas exigencias de los currículos, el número, la complejidad y diversidad de los y las estudiantes, las altas tasas de absentismo y abandono escolar prematuro, la aparición de dificultades de convivencia y la disminución de la disponibilidad de las familias para la educación de sus hijos e hijas, obligan a una acción colaboradora y complementaria de estos y estas profesionales junto con el profesorado (Castillo, 2012; Hernández y Sancho, 2004; March y Orte, 2014; Parcerisa, 2008). Asimismo, la ubicación de los centros escolares en contextos donde existen múltiples recursos que pueden ser aprovechados en beneficio de la comunidad educativa, es otra variable que apoya la idea de que hay que establecer redes de apoyo entre todos los colectivos que, de forma directa o indirecta, están relacionados con la educación (López, 2013). En definitiva, coincidimos con Cuesta, Martínez, Cuesta, Sánchez y Orozco (2017:148) cuando apuntan que "la presencia de educadores sociales en el contexto educativo proporcionaría una nueva manera de contemplar la educación, asumiendo un conjunto de competencias especializadas relacionadas con el seguimiento, cercanía, comunicación o la mediación, aspectos necesarios para lograr dar respuesta a las demandas educativas actuales".

En el año 2004, los colegios profesionales de educadores y educadoras sociales hicieron público un documento titulado El educador y la educadora social en el Estado español: una concreción de su trabajo en centros escolares (ASEDES, 2004). Este documento respondía a una realidad: en diversas zonas de España se empezaba a introducir este profesional en escuelas e institutos de educación secundaria. Todos los argumentos expuestos anteriormente explican la elaboración de este documento.

Como profesión reciente, la educación social se ha ido desarrollando, aportando mayor definición a sus ámbitos y funciones, con el objeto de dar respuesta a nuevas necesidades socioeducativas (Amador, Cardena, Esteban y Terrón, 2014), desarrollo fundamentalmente motivado por dos factores: la crisis de la escuela y la idea del estado de bienestar. No podemos negar que el ambiente social en el que se sitúa la profesión de educación social es, necesariamente, dinámico, vivo, a la vez que variado y complejo, lo que explica la aparición de nuevos espacios profesionales. Así pues, las nuevas necesidades de la sociedad han planteado nuevos retos a la educación social. 


\section{EDUCACIÓN SOCIAL EN LA ESCUELA: ESTADO DE LA CUESTIÓN EN ESPAÑA}

Poco a poco, el ejercicio de la educación social en los centros educativos de España va tomando forma y adoptando diferentes alternativas según nuestro sistema constitucional de distribución de competencias. López (2013) plantea tres modelos de acción diferentes según las Comunidades Autónomas (CCAA), que definen la labor de los y las educadores sociales en Ios centros escolares: a) educadores y educadoras sociales adscritos a los centros escolares de forma institucional, presente en Andalucía, Extremadura y Castilla-La Mancha, en el que este/a profesional forma parte de los equipos de los centros escolares, prioritariamente de secundaria; b) educadores y educadoras sociales pertenecientes a los Servicios Sociales municipales, en comunidades como Baleares, Euskadi, Galicia, Madrid o Barcelona; y, c) proyectos concretos liderados por educadores y educadoras sociales en los centros escolares que sería el modelo seguido en el resto de CC.AA.

Este último modelo cristalizó en la planificación de proyectos de intervención en los centros escolares coordinados por educadores y educadoras sociales, mediante la firma previa de convenios entre los Ayuntamientos y los gobiernos autonómicos, o bien de los ayuntamientos directamente. Más recientemente ha evolucionado a otras modalidades de integración de la educación social en centros docentes, también a través de entidades intermediarias, pero no sólo bajo la forma jurídica de convenio. Se trataría de las iniciativas que actualmente se están implantando en las comunidades de Balears y Canarias.

Las Illes Balears recupera, a través de las Consejerías de Servicios Sociales y de Educación y para el curso 2017-2018, un programa experimental, que ya se había puesto en marcha en el año 2009 y que se suprimiera en el 2011 a pesar de su valoración positiva. Esta iniciativa supone la incorporación de educadores y educadoras sociales en diez centros de educación secundaria en el marco del Programa para la Mejora de Convivencia. Las funciones previstas serían prevenir conflictos en los centros, evitar el fracaso, abandono y absentismo escolar, promover la convivencia y atender a las dificultades sociales del alumnado. La fórmula empleada para cubrir estas plazas (y ahí está la novedad) reside en que la contratación se realiza a través de una entidad adjudicataria hasta junio de 2018 sujeta a una serie de obligaciones -organización, gestión e implementación del programa-, por lo que los educadores/as contratados/as no dependerán directamente de la Administración Pública. 
Por su parte, en la Comunidad Autónoma de Canarias está a punto de implementarse otra alternativa en convenio con el Colegio Profesional. La Resolución de 26 de septiembre de 2017 publica un convenio de colaboración entre la Consejería de Educación y Universidades y el Colegio Profesional de Educadores y Educadoras Sociales de Canarias (CEESCAN) para la mejora del sistema educativo y la educación social. A través de este convenio se manifiesta la "voluntad de iniciar y aunar esfuerzos en la promoción de la Educación Social en todas las facetas del sistema educativo", y se plantea una experiencia piloto experimental en el curso 2017/2018, integrando 36 educadores y educadoras en centros educativos seleccionados por la administración educativa. Las peculiaridades de este modelo es que se relacionan y dependen exclusivamente del Colegio Profesional, no dando lugar a relación laboral ni administrativa con la Consejería de Educación y Universidades, siendo el CEESCAN quien debe facilitar este personal técnico, así como la dirección, coordinación y supervisión del mismo. En el momento que estamos escribiendo, ya fueron publicadas las listas de personas admitidas para la selección de educadores/as sociales del proyecto piloto de Educación Social Escolar, Comunitaria y para la Convivencia (ESEC). Además, el convenio recoge que, desde la educación social, se desarrollarán y plantearán en el centro educativo "propuestas de mediación familiar, resolución de conflictos, reducción del absentismo escolar, fomento de la convivencia, prevención del acoso escolar, atención a la diversidad y desarrollo comunitario y participado", tendrán la consideración de personal no docente y realizarán sus actuaciones "en horario y ubicación extraescolar".

Detengamos ahora en las experiencias de integración del educador o educadora social de forma institucional en la escuela. El proceso que institucionaliza la figura del educador y de la educadora social en los centros escolares se inició en año 2002, en las comunidades autónomas de CastillaLa Mancha y Extremadura.

La Orden de 26 de junio de 2002 de la Consejería de Educación y Cultura de Castilla-La Mancha desarrolla medidas previstas en el Plan de Mejora de la Educación Secundaria Obligatoria, estableció que debía dotarse a los centros, que lo justificasen suficientemente por la problemática de convivencia, de un educador o educadora social para que desarrollara tareas de mediación y control en colaboración con las familias y con otras instituciones. Asimismo, debería desarrollar medidas para asegurar la asistencia regular del alumnado a las clases, actuando en aquellas situaciones de alto riesgo con acciones positivas de control y prevención del absentismo escolar. Este profesional intervendría bajo la dependencia del equipo directivo y en coordinación con los/as tutores/as y el equipo de orientación, situándose en el departamento de este último equipo. Su incorporación efectiva "se producirá a principios del año 2003" (Galán, Castillo y Pellissa, 2012, p. 14), con la 
contratación de 22 educadores/as sociales, como personal funcionario de administración general, A2, escala técnico sociosanitaria, en algunos centros de educación secundaria (Galán y Castillo, 2008). A fecha 6 de junio de 2013, "únicamente se encuentra la figura del/a Educador/a Social en 40 IES de un total de 227 que existen en toda la región" (CGCEES, 2015:20). A estos y estas profesionales habría que añadir 14 plazas convocadas mediante procedimiento de concurso-oposición para el año 2017.

El mismo año, el 25 de octubre, unas Instrucciones de la Dirección General de Ordenación, Renovación y Centros de la Junta de Extremadura, establecen las funciones y ámbitos de actuación de los educadores y educadoras sociales en centros de educación secundaria de la red pública, basándose en la realidad compleja de los centros, y las situaciones problemáticas que en ellos pueden darse y reconociéndoles como profesionales que pueden prevenirlas y buscar soluciones en colaboración con los restantes miembros de la comunidad educativa. El/la educador/a social se integra en el departamento de orientación y colabora con el departamento de actividades complementarias y extraescolares. Su incorporación a los centros educativos se produjo entre septiembre y octubre de 2002, y "supuso contratar a 118 Educadores Sociales diplomados" mediante una bolsa de trabajo (Galán y Castillo, 2008, p. 121), un educador o educadora social por cada instituto de educación secundaria. Inicialmente fueron considerados personal de administración y servicios, hasta que con la promulgación de la Ley 4/2011 de educación de Extremadura, pasaron a tener la consideración de agentes educativos no docentes -grupo II del personal laboral-. En convocatorias posteriores, y para consolidar estos puestos de trabajo se convocaron pruebas selectivas por el sistema de concurso-oposición para cubrir 155 plazas en el año 2006, 30 en el 2010 y 18 en el 2013 (253 educadores y educadoras sociales están ejerciendo labores en centros educativos de Extremadura).

Por su parte, en Andalucía no fue hasta la entrada en vigor del Decreto 19/2007 cuando se introduce la figura profesional del educador y de la educadora social con categoría de funcionariado adscrita a los equipos de orientación educativa que atiendan a alumnado que presente una especial problemática de convivencia escolar. No obstante, la incorporación de la figura profesional del educador y educadora social en los centros educativos en esta comunidad autónoma ya se había producido anteriormente por Resolución de 16 de octubre de 2006, por la que se realiza convocatoria para la cobertura provisional durante el curso 2006-2007 en puestos de educación social en el ámbito educativo. Como consecuencia de la entrada en vigor de la Ley 17/2007, de educación de Andalucía, en septiembre de 2010 se dictaron las Instrucciones que regulan la intervención del/a educador/a social en el ámbito escolar como personal laboral del grupo II, planteándose su incorporación no sólo en el departamento de orientación de secundaria, sino también en 
los equipos de orientación educativa, en el caso de la educación infantil y primaria. En relación con las funciones, parece que "convivencia, participación e interculturalidad aparecen como competencias fundamentales para el desarrollo del perfil profesional de los educadores (...) se trata de muchas y variadas funciones, de las que, en unos centros se hace más énfasis en unas u otras en relación a las necesidades, las prioridades y los contextos" (Sierra et al., 2017: 488). Según datos de CGCEES (2015), en el curso 2014/15, la plantilla de los 150 equipos que componen la red "ha sido de 695 orientadoras/es, 147 maestras/os de audición y lenguaje, 50 maestras/ os de E.O.E. (educación compensatoria), 130 médicas/os y 59 Educadoras/ es" (p. 22). A estos datos habría que añadir cinco plazas que han salido publicadas en la Oferta de Empleo Público de 2017.

En consecuencia, en las tres comunidades descritas, la incorporación de la figura profesional del educador y educadora social a los centros educativos, por parte de las administraciones autonómicas, adoptó modelos sustancialmente diferentes en cuanto al formato de incorporación y al número de profesionales integrados: personal educativo no docente en todos los centros de secundaria en Extremadura, funcionario/a en los departamentos de orientación en institutos de educación secundaria con problemas de convivencia en Castilla-La Mancha; y, un/a miembro en estos departamentos en secundaria o en los equipos de orientación educativa en infantil y primaria, también en centros con dificultades en su convivencia, en Andalucía, aunque finalmente sólo ha incorporado profesionales de la Educación Social a los equipos de orientación educativa. Sus funciones principales, responden a exigencias derivadas del absentismo escolar, problemas de convivencia, mediación, prevención...

A continuación, haremos referencia a otros modelos de integración, no dependientes directamente de la administración autonómica, seguidos en diferentes comunidades, en los que la educación social entra en los centros educativos sin que sus profesionales formen parte del personal de estos. No pocas son las experiencias que podemos citar, ya que ésta ha sido la forma mayoritaria adoptada en España; por citar algunos ejemplos nos referiremos a dos experiencias. En Aragón, los educadores y educadoras sociales forman parte del Proyecto de Integración de Espacios Escolares en centros de cualquier nivel no universitario, y dirigen su intervención a estudiantes con dificultades de integración social. En Euskadi su acción se implementa desde las «comunidades de aprendizaje» y está dirigida a la dinamización comunitaria y escolar, la implicación y participación de la familia, así como a la observación social. Para quienes deseen conocer experiencias que se están Ilevando a cabo en otras zonas de España, recomendamos la consulta 
de los siguientes textos: Arrikaberri y cols. (2013), ASEDES (2004), CastiIlo y Bretones (2014), Castillo, Galán y Pellissa (2012), CGCEES (2015) y Parcerisa (2008).

Finalmente, nos detendremos en Galicia por ser una Comunidad Autónoma en la que no está incorporada formalmente la figura profesional del educador o educadora social en la escuela; $y$, porque se ha tomado como referente para el estudio comparativo que recogemos en el siguiente apartado.

En primer lugar, la entrada de los y las profesionales de la Educación Social en el ámbito escolar se inicia bajo la modalidad de «docente en educación secundaria». De forma más específica, los/as titulados en Educación Social, diplomatura o grado, pueden acceder a los cuerpos de Profesores de Enseñanza Secundaria y de Formación Profesional; en el primero se integran las especialidades de «Formación y Orientación Laboral» y de «Intervención Sociocomunitaria» y en cuanto al Cuerpo de Profesores de Formación Profesional, las especialidades son las que pertenecen a la familia profesional de «Servicios socioculturales y a la comunidad». En segundo lugar, además de la docencia, los y las educadoras sociales se integran tomando el modelo de educador/animador/monitor(a) en las actividades extraescolares que se desarrollan en los centros escolares, por iniciativa del propio centro, de las AMPAs o incluso desde los ayuntamientos. Son opciones claramente desiguales; en la primera se trata de plazas a las que se accede a través de la Oferta de Empleo Público, de carácter más estable, a tiempo completo y con reconocimiento acorde a su titulación universitaria, aunque con función únicamente docente. La segunda opción estaría definida, generalmente, por unos parámetros diferentes: el tipo de contrato (si existe) tiene carácter eventual, a tiempo parcial y, con frecuencia, sin reconocimiento laboral ni económico acorde a su titulación universitaria, pero sí hacen intervención social, en concreto, en animación sociocultural.

Por otra parte, y dependientes directamente de la Consejería de Educación, la presencia directa de educadores y educadoras sociales en los centros de secundaria podría haberse realizado, pero no ha sido efectiva, con su incorporación a los Equipos de Orientación Específicos (EOEs). La normativa que los regula (Decreto 120/1998) en el artículo 5, establece su composición, incluyendo un trabajador y/o educador social. A pesar de este reconocimiento legal, la práctica revela que en ninguno de los EOEs existentes en la actualidad en Galicia (uno por provincia), se cubrió la plaza con algún/a educador/a social. Según datos de la administración, de fecha 30-10-2017, en dos están cubiertas con trabajadores/as sociales y en los otros dos están vacantes. 
Otra opción seguida en Galicia, es aquella que la situaría entre las comunidades en las que la integración de los y educadores y educadoras sociales se vincula a los Servicios Sociales municipales. Cuando asume las competencias en servicios sociales e incorpora a los educadores y educadoras sociales a sus equipos multidisciplinares de trabajo, incorpora la educación social al ámbito escolar, a partir del diseño ad hoc de proyectos. Según el Colegio de Educadoras y Educadores Sociales de Galicia (CGCEES, 2015) se está realizando un trabajo en contacto directo con las escuelas y los institutos de secundaria, a través de los Servicios Sociales Comunitarios. El Colegio Oficial tiene constituido un grupo de trabajo -aBeira- en el que educadores/ as sociales de ayuntamientos que ejecutan programas de prevención e intervención en las escuelas e institutos de sus municipios, intercambian sus experiencias. Su trabajo está orientado a la lucha contra el absentismo escolar, prevención de conflictos, educación afectivo-sexual..., y se concreta en programa tales como Dorna: Embárcate en la salud, De buen rollo: programa de habilidades para la vida, Proyecto de intervención socioeducativa en los institutos del barrio de Labañon o Programa Conecta Aleida.

\section{ESTUDIO COMPARATIVO DE LOS PLANES DE ESTUDIO DE GRADO EN EDUCACIÓN SOCIAL DE LAS UNIVERSIDADES GALLEGAS Y EXTREMEÑA}

Este apartado se enmarca en el ámbito de la formación universitaria de los graduados y graduadas en Educación Social. Nos detendremos en analizar qué formación inicial reciben relativa a la educación social en la escuela, qué tendencias están marcando su trayectoria, a partir del análisis de los planes de estudio conducentes a la obtención del título de «Grado en Educación Social». En relación con la formación postgrado, hemos de decir que no identificamos ningún máster que abordara directamente este ámbito de intervención.

Para ello hemos elegido los planes formativos de cuatro universidades españolas. Se trata de una muestra intencionada en las universidades elegidas: las tres universidades que ofertan Educación Social; y la de Extremadura, por tratarse de una comunidad con una dilatada experiencia en la incorporación de los educadores sociales en la escuela (desde el año 2002). Para cada una de las universidades seleccionadas se han examinado las Memorias de Grado, así como los planes de estudio de Grado vigentes publicados en el BOE. 
La metodología seguida responde a un análisis de contenido de: las competencias recogidas en las memorias de grado; y las materias existentes en los Grados en Educación Social de las cuatro universidades, buscando su vinculación con el perfil profesional de la educación social en la escuela.

\subsection{Análisis de competencias}

Uno de los cambios que produjo la adaptación del sistema universitario al Espacio Europeo de Educación Superior, tuvo que ver con las competencias, entendidas como capacidades y destrezas vinculadas con la profesión que deben alcanzar los y las estudiantes una vez finalicen los estudios.

Si las competencias, son los resultados esperados una vez finalicen los y las estudiantes su titulación, nos ha parecido de interés partir de su estudio. Para empezar este análisis procederemos a la revisión de competencias relacionadas con la escuela que figuran en el Libro Blanco «Título de Grado en Pedagogía y Educación Social» para el Grado de Educación Social (ANECA; 2005). De las 26 competencias específicas, ninguna de ellas hace mención explícita al ámbito escolar, a la educación formal o al sistema educativo; aunque no es un ámbito que se manifieste de forma clara, puede entreverse en diferentes competencias que aluden a «ámbitos de actuación», «intervención socioeducativa», "procesos socioeducativos».

Centrándonos en las Memorias de Grado en Educación Social de la universidad extremeña y de las gallegas, las competencias estrictamente referidas al ámbito escolar son también inexistentes, constando un alto paralelismo con las propuestas en el Libro Blanco. Nuevamente, por tanto, las alusiones son indirectas, en ningún caso se menciona de forma expresa «escuela», como ámbito profesional o de intervención.

En síntesis, podemos concluir que la tendencia que marcó la elaboración de las competencias en el Grado de Educación viene definida por no hacer patente el marco escolar, dejando, por el contrario, más explícitos otros ámbitos clásicos de la Educación Social.

\subsection{Análisis de materias}

Esta sección responde al análisis de los planes de estudio vigentes en las cuatro universidades (Tabla 1 ), respecto a las materias específicamente orientadas al ámbito de la escuela. Como puede apreciarse, las materias son escasas, si atendemos a aquellas que de forma explícita y literal utilizan en su denominación el vocablo «escuela», "educación formal» o «sistema educativo». Solamente en dos de las cuatro universidades hemos identificado una materia: Universidad de Santiago compartida con otros ámbitos (familia 
y comunidad) y Vigo, referida únicamente al sistema educativo. En ambos planes de estudio, estas materias son de carácter obligatorio, se imparten en segundo curso y tienen asignados 6 créditos ECTS. El peso porcentual que representa esta formación es del 2,5\%.

\section{TABLA 1}

Asignaturas en los planes de Estudio de Grado en Educación Social relativas a la escuela

\begin{tabular}{|l|c|c|c|c|}
\hline \multicolumn{1}{|c|}{ Universidad } & Materias & Carácter & $\begin{array}{c}\text { Créditos } \\
\text { ECTS }\end{array}$ & Curso- \\
\hline A Coruña & Ninguna & - & - & - \\
\hline $\begin{array}{l}\text { Santiago de Com- } \\
\text { postela }\end{array}$ & $\begin{array}{c}\text { Familia, escuela y comunidad } \\
\text { en la educación social }\end{array}$ & Obligatoria & 6 & $2^{\circ}$ \\
\hline Vigo & $\begin{array}{c}\text { Educación social en el sistema } \\
\text { educativo }\end{array}$ & Obligatoria & 6 & $2^{\circ}$ \\
\hline Extremadura & Ninguna & - & - & - \\
\hline
\end{tabular}

Fuente: Elaboración propia a partir de BOEs.

Análisis de convocatorias públicas de educación social en el ámbito escolar: el caso de Extremadura.

Diferentes autores han centrado sus reflexiones en las funciones y competencias de los/as educadores/as sociales en los centros educativos (Arrikaberri y cols., 2013; ASEDES, 2004; Cuesta et al, 2017; González et al., 2016; Galán, 2008; Galán y Castillo, 2008; Merino, 2013; Vega, 2013), coincidiendo en su importancia así como en la amplitud de las mismas.

Concretamente, González, Olmos y Serrate (2016) en un estudio muy reciente realizado a una muestra de 440 educadores y educadoras sociales, concluyeron que el/la educador/a social desempeña funciones relativas a la atención de situaciones conflictivas o de necesidad (más que otros/as profesionales de los centros) y su trabajo educativo se dirige a intervenciones de prevención del absentismo escolar y control del alumnado a la Ilegada al centro escolar, tareas de detección y prevención de factores de riesgo, escuelas de padres y programas de información, mediación en conflictos de convivencia, desarrollo de programas de comunicación, asesoramiento y apoyo socioeducativo a la comunidad educativa y planificación de ofertas relacionadas con servicios culturales.

Seguidamente, y para establecer las funciones y ámbitos de actuación de los/as educadores/as sociales en centros de educación secundaria de Extremadura, nos detendremos en el análisis de distintos documentos que los regulan, así como en las últimas órdenes publicadas convocando pruebas selectivas. 
Centrándonos en las Instrucciones que regulan las funciones de los educadores y educadoras sociales en los centros de educación secundaria de Extremadura, podemos diferenciar, por un lado, aquellas funciones más relacionadas con la gestión y organización, como participación en la elaboración de documentos escolares -Proyecto Educativo de Centro (PEC), Plan de Acción Tutorial (PAT)...-, relaciones con otros centros y familias...; y, por otro lado, aquellas otras que implican una acción más educativa, denominados por Galán (2008) «programas socioeducativos»: educación para la salud, ocio y tiempo libre, educación para la convivencia, absentismo escolar, modificación de conducta, desarrollo de habilidades, fomento de la igualdad y resolución de conflictos y mediación. Además, comparte responsabilidades y funciones con el Departamento de Orientación en el desarrollo del Plan de Orientación Académico y Profesional (POAP), y con el de Actividades Complementarias y Extraescolares en la Programación de Actividades Culturales y Deportivas; $y$, finalmente, permiten que cada centro, según sus necesidades, les puedan encomendar otras funciones.

Coincidimos con Galán y Castillo (2008), al considerar que las funciones que la Administración asignó a los educadores y educadoras sociales son poco específicas, calificándolas de "demanda desmesurada y dispersa". Posteriores Instrucciones ponen mayor precisión a las funciones inicialmente asignadas. Así, por ejemplo, las Instrucciones de Junio de 2006, en los artículos 161 a 164, establecen que los/as educadores sociales se ocuparán de la prevención de situaciones educativas desfavorables, la mediación, la prevención y control del absentismo escolar, el desarrollo de habilidades sociales y el control sobre la permanencia de los estudiantes de la ESO en el centro, así como el transporte escolar y la prevención y/o atención de situaciones de violencia de género. En relación al absentismo escolar el Decreto 142/2005, así como la Orden de 19 de diciembre de 2005, también ayuda a clarificar las funciones, al incluir a los y las profesionales de la Educación Social en la composición de las Comisiones de Absentismo de los centros. En último lugar, en Decreto 50/2007, se incluye al/a educador/a social como miembro de la Comisión de Convivencia asignándole el papel de mediador para la resolución de conflictos de convivencia.

Atenderemos seguidamente a las últimas convocatorias de pruebas selectivas para personal laboral de la Comunidad Autónoma de Extremadura (Orden de 27 de diciembre de 2013, convocatoria oposiciones, y Orden de 20 de abril de 2017, lista de espera), y más concretamente al análisis del temario específico de «educadores» recogido en las mismas.

Llama la atención, en primer lugar, la denominación de la categoría/ especialidad: no figura «educador social», sino «educador», aunque la titulación requerida sea «Diplomatura en Educación Social o Grado equivalente». En 
relación al temario, de los 72 temas incluidos, la mayoría hacen referencia a líneas profesionales de los/as educadores/as sociales: educación para la salud, drogodependencias, educación sexual, igualdad, menores (protección y reforma), resolución de conflictos, atención a la diversidad, mediación, inmigración...; un segundo bloque, de menor entidad, toma su base en la psicología (psicología del desarrollo, motivación, autoestima,...); y, finalmente, un tercer bloque de contenidos específicos del mundo escolar (Plan de Convivencia, PEC, POAP, PAT, órganos colegiados, órganos de coordinación docente, derechos y obligaciones del alumnado, absentismo escolar,...).

Así mismo debemos resaltar pequeños cambios en el temario incluido en cada una de las convocatorias. En la convocatoria más reciente, se suprimieron temas referidos a cuestiones más teóricas y científicas de alguna de las líneas señaladas anteriormente, como por ejemplo: «Educación Formal y No Formal», "Análisis histórico de los sistemas de protección de menores en España», "Socialización y aprendizaje. La escuela como institución socializadora» o «Animación Sociocultural: nacimiento y evolución. Concepto de animación sociocultural, objetivos y funciones». Por el contrario, se incluyeron temas relacionados con problemáticas muy actuales como «Educación emocional. Estrategias y programas de intervención», «El fracaso escolar. Causas y consecuencias. Medidas para la prevención e intervención», «Innovación educativa», "Orientación profesional en los centros educativos desde el POAP», «Servicio del transporte escolar. Funciones y competencias del educador social».

\section{CONCLUSIONES}

Sumándonos a lo afirmado por Castillo (2012), la incorporación de la Educación Social a la escuela se ha producido de forma dispar en España, tanto si tenemos en cuenta los aspectos contractuales, como la propuesta y el desarrollo normativo de las funciones previstas en cada comunidad autónoma.

En cuanto a los aspectos contractuales, en aquellas CC.AA. en las que su incorporación se ha hecho de una forma más institucional, en dos de ellas el educador o educadora social forma parte del personal laboral-, mientras que en Castilla-La Mancha es considerado/a personal funcionario de la escala técnico sociosanitaria. En el resto de iniciativas, se regula por la legislación vigente que afecta al empleo privado.

Aunque esta incorporación, en muchos casos, se ha reducido a experiencias territoriales o a determinados centros con especiales necesidades, principalmente de secundaria, evidenciamos un aumento de Comunidades 
Autónomas que introducen educadores y educadoras sociales en los centros escolares, como han sido las iniciativas emprendidas por las Illes Balears y Canarias.

La evolución de las plazas convocadas en las tres Comunidades Autónomas que tienen como personal propio a educadores y educadoras sociales que realizan sus tareas en centros educativos, ha sido desigual. Sólo Extremadura mantiene una periodicidad aproximada de 4 años en las convocatorias de plazas -muy inferior a la inicial-; en las otras dos CC.AA. han tenido que pasar entre 8-9 años para una nueva convocatoria con una merma significativa en el número de plazas ofertadas.

Refiriéndonos a las funciones, existe tal dispersión, que creemos necesario que se realice una descripción de este puesto de trabajo -tareas y responsabilidades que conforman el mismo- entre los Colegios Profesionales de Educadores y Educadoras Sociales y las Administraciones Educativas.

Por último, nos hemos preguntado: ¿la formación inicial de los y las educadores sociales da respuesta a tan ambiciosa demanda?, ¿siendo las materias específicas tan exiguas, es posible que otras materias puedan completarla?, ¿podemos hablar de una formación transversal que pueda ser aplicada en distintos contextos, incluido el escolar, pero no exclusiva de éste?

Las respuestas a estas cuestiones no quedan totalmente claras. Por una parte, se puede afirmar que la formación inicial de los/as educadores/as sociales, a partir de los grados, incluye preparación en el trabajo socioeducativo en muchos de los ámbitos exigidos, incorporando materias determinadas para cada uno de ellos (atención a la diversidad, educación familiar, mediación, animación sociocultural, menores, educación sexual, para la salud y drogodependencias,...). Se trata de una preparación que está presente, aunque faltaría por comprobar si en su tratamiento hay una adecuación definida a las peculiaridades de la intervención en el marco escolar.

Por otra parte, lo que no parece tan claro, es que el educador y la educadora social, tenga formación propia en cuanto a estructura, organización y funcionamiento de los centros escolares, si no ha cursado ninguna materia sobre este contenido. Probablemente, esta realidad nos haga plantear la oportunidad de consultar a los/as educadores/as sociales en ejercicio si han sentido la necesidad de incorporar contenidos que les permitan acercarse a las singularidades de las dinámicas del sistema educativo actual. Conocer qué es y cómo se elabora un PEC, un Plan de Convivencia, un PAT... son propuestas de trabajo obligatorias en los centros educativos que un/a 
educador/a social debiera conocer si va a trabajar en este ámbito. El propósito final sería optimizar las aportaciones que la Educación Social puede hacer a la institución escolar.

Pero, si estas preguntas las ampliamos a las otras posibilidades de incorporación de la Educación social a la escuela -profesor/a, educador/ animador/monitor(a) en las actividades extraescolares, o educador/educadora social perteneciente (o en colaboración) a los Servicios Sociales municipales que realiza proyectos ad hoc en centros educativos-, podemos afirmar que su formación sería más que pertinente para llevar a cabo sus intervenciones profesionales puesto que sus planes de estudios incorporan materias bien relacionadas con las titulaciones incluidas en la familia profesional «Servicios Socioculturales y a la Comunidad» y «Formación y Orientación Laboral»-inserción sociolaboral, intervención socioeducativa con personas con diversidad funcional, animación sociolaboral,...-, bien materias en las que se enmarcan la planificación y el desarrollo de las actividades extraescolares -Animación Sociocultural y Pedagogía del Ocio-. Quedaría menos abordada su formación en temáticas específicas relativas al rendimiento académico y al fracaso escolar.

Para finalizar, compartimos con López (2013) que "necesitamos profesionales bien preparados, con formación específica, que posibiliten que cada profesional pueda hacer su trabajo. Las educadoras y educadores sociales, por su formación en el ámbito socioeducativo y cultural, aportan y pueden aportar mejoras al sistema educativo y sobre todo a la cobertura de las necesidades de nuestro alumnado y de sus familias", pero para que esto sea una realidad no debemos olvidar su formación, sino que debemos empeñarnos en que ésta responda a las exigencias y necesidades de la práctica.

\section{BIBLIOGRAFÍA}

Amador, LV.; Esteban, M.; Cárdenas, R. y Terrón, T. (2014). Ámbitos de profesionalización del educador/a social: perspectivas y complejidad. Revista de Humanidades, 21, 3. http://www.revistadehumanidades.com/articulos/47-ambitos-de-profesionalizaciondel-educador-a-social-perspectivas-y-complejidad

ANECA (2005). Libro Blanco del Título de Grado en Pedagogía y Educación Social I. Madrid: Agencia Nacional de Evaluación de la Calidad y Acreditación.

Arrikaberri, M.; Caballero, M.; Huarte, J.; Tanco, C.; Biurrum, A.; Etayo, Y. y Urdániz, S. (2013). Educadoras y educadores sociales en los centros educativos. Hacia una integración orgánica y funcional en la Comunidad Foral de Navarra. RES Revista de Educación Social, 16, 1-17. 
ASEDES (2004). El educador y la educadora social en el estado español: una concreción de su trabajo en centros escolares. http://www.eduso.net/archivos/ESCE.doc

ASEDES (2007): Catálogo de Funciones y Competencias del educador y la educadora social. Barcelona: Grafox. http://www.eduso.net/archivo/docdow.php?id=143

Castillo, M. (2012). La intervención de los educadores y educadoras sociales en la escuela: limitaciones, retos y perspectivas de futuro. Educación Social. Revista de Intervención Socioeducativa, 51, 133-151.

Castillo, M. y Bretones, E. (2014). Acción social y educativa en contextos escolares. Barcelona: UOC.

CGCEES (2015). La Educación Social en el estado español: la profesión educativa emergente dentro y fuera del ámbito académico. http://ceesg.org/users/ceesg/obxetos/cgcees-es-en-centros-educativos-noviembre-2015.pdf

Chozas, A. (2003). El educador social en las instituciones educativas: expectativas y tareas. En J. García Molina (Coord.). De nuevo, la Educación Social (pp. 127-35). Madrid: Dykinson.

Cuesta, M.C.; Martínez, M.A.; Cuesta, J.L.; Sánchez, S., y Orozco, M.L. (2017). El educador social en la enseñanza secundaria. La mediación escolar como alternativa a la resolución de conflictos. Ehquidad International Welfare Policies and Social Work Journal, 7, 145-174.

Decreto 120/1998, de 23 de abril, por el que se regula la orientación educativa y profesional en la Comunidad Autónoma de Galicia. Diario Oficial de Galicia, 79 de 27 de abril de 1998.

Decreto 19/2007, de 23 de enero, por el que se adoptan medidas para la promoción de la Cultura de Paz y la Mejora de la Convivencia en los Centros Educativos sostenidos con fondos públicos. Boletín Oficial de la Junta de Andalucía, 25 de 2 de febrero de 2007.

Decreto 142/2005, de 7 de junio, por el que se regula la prevención, control y seguimiento del absentismo escolar en la Comunidad Autónoma de Extremadura, 68 de 14 de Junio de 2005

Decreto 50/2007, de 20 de marzo, por el que se establecen los derechos y deberes del alumnado y normas de convivencia en los centros docentes sostenidos con fondos públicos de la Comunidad Autónoma de Extremadura, Diario Oficial de Extremadura, 36 de 27 de Marzo de 2007.

Esteban, M. y Amador, L. (2017). La Educación Ambiental como ámbito emergente de la Educación Social. Un nuevo campo socioambiental global. RES, Revista de Educación Social, 25, 134-147.

Froufe Quintas, S. (1997). Los ámbitos de intervención en la Educación Social. Aula, 9, 179-200.

Galán, D. (2008). Los educadores sociales en los centros de Educación Secundaria de Extremadura. Pedagogía Social. Revista Interuniversitaria, 15, 57-70.

Galán, D. y Castillo, M. (2008). El papel de los educadores sociales en los centros de secundaria: una propuesta para el debate. Educación Social. Revista de Intervención Socioeducativa, 38, 121-133.

Galán, D.; Castillo, M. y Pellissa, B. (2012): La incorporació dels educadors socials a l'escola: l'experiència present $i$ les perspectives de futur. Barcelona: UOC. 
Gómez Serra, M. (2003). Aproximación conceptual a los sectores y ámbitos de intervención de la educación social. Revista Interuniversitaria de Pedagogía Social, 10, 233-251.

González, M.; Olmos, S. y Serrate, S. (2016). Análisis de la práctica profesional del educador social en centros de educación secundaria. Pedagogía Social. Revista Interuniversitaria, 28, 229-243.

Hernández, F. y Sancho, J.M. (2004). El clima escolar en los centros de secundaria: más allá de los tópicos. Madrid: MEC-CIDE.

Instrucciones de 25 de octubre de 2002, de la Dirección General de Ordenación, Renovación y Centros por las que se establecen las funciones y ámbitos de actuación de los educadores sociales en centros de educación secundaria de la red pública dependiente de la Consejería de Educación, Ciencia y Tecnología de la Junta de Extremadura. http://jcpintoes.en.eresmas.com/instrueducsoc.pdf

Instrucciones de 27 de junio de 2006, de la Dirección General de Política Educativa, por la que se concretan las normas de carácter general a las que deben adecuar su organización y funcionamiento los Institutos de Educación Secundaria y los Institutos de Educación Secundaria Obligatoria de Extremadura. file:///D:/Users/Admin/Desktop/Ed\%20 Social\%20en\%20la\%20escuela/legislacion\%20 extremadura/Inst_ES_270606.pdf

Instrucciones de 17 de septiembre de 2010, de la Dirección General de Participación e Innovación Educativa de la Consejería de Educación de la Junta de Andalucía, por las cuales se regula la intervención del educador y educadora social en el ámbito educativo. http://www.adideandalucia.es/normas/instruc/Instruc17sept2010educadorsocial.pdf

Ley 17/2007, de educación de Andalucía. Boletín Oficial de la Junta de Andalucía, nº 252, de 26 de diciembre de 2007.

Ley 4/2011, de 7 de marzo, de educación de Extremadura. Diario Oficial de Extremadura, ${ }^{\circ}$ 47 de 9 de marzo de 2011.

Longás Mayayo, J. (2000). Educación Social y escuela, nuevos ámbitos de intervención. Educación Social. Revista de Intervención Socioeducativa, 15, 101-106.

López, R. (2013). Las educadoras y los educadores sociales en centros escolares en el estado español. RES. Revista de Educación Social. 16. http://www.eduso.net/res/pdf/16/ jor_res_\%2016.pdf

March, M.X. y Orte, C. (2002). La escuela como espacio de la Pedagogía Social. En J. Ortega (Coord.). Nuevos retos de la Pedagogía Social: la formación del profesorado (pp. 322-329). Salamanca: Sociedad Ibérica de Pedagogía Social.

March, M.X. y Orte, C. (Coords) (2014). La pedagogía social y la escuela. Los retos socioeducativos de la situación escolar en el s. XXI. Barcelona: Octaedro.

Merino, R. (2013). La educación social en la escuela/la escuela en la educación social. RES Revista de Educación Social, 16, 1-9.

Molina, J.G. y Blázquez, A. (2015). El educador social en la educación secundaria. Educación Social. Revista de Intervención Socioeducativa, 32, 39-59.

Orden de 26 de junio de 2002 de la Consejería de Educación y Cultura por la que se desarrollan determinadas medidas contempladas en el Plan para la Mejora de la Educación Secundaria Obligatoria en Castilla-La Mancha. Diario Oficial de Castilla-La Mancha, 78 de 26 de junio de 2002. 
Orden de 19 de diciembre de 2005 por la que se regulan las actuaciones de la Consejería de Educación para la prevención, control y seguimiento del absentismo escolar. Diario Oficial de Extremadura, 2 de 5 de enero de 2006.

Orden de 27 de diciembre de 2013 por la que se convocan pruebas selectivas para el acceso a puestos vacantes del Grupo II de personal laboral de la Administración de la Comunidad Autónoma de Extremadura. Diario Oficial de Extremadura, 249 de 30 de diciembre de 2013.

Orden de 20 de abril de 2017 por la que se convocan pruebas selectivas para la constitución de listas de espera en el Cuerpo Técnico/Grupo II Categoría Educador de la Administración de la Comunidad Autónoma de Extremadura. Diario Oficial de Extremadura, 77 de 24 de abril de 2017.

Ortega, J. (2005). Pedagogía Social y Pedagogía Escolar. La Educación Social en la escuela. Revista de Educación, 336, 111-127.

Ortega, J. (2014). Educación Social y enseñanza: Ios educadores sociales en los centros educativos, funciones y modelos. EDETANIA, 45, 11-31.

Parcerisa, A. (2008). Educación Social en y con la institución escolar. Revista Interuniversitaria de Pedagogía Social, 15, 15-27.

Pérez Alonso-Geta, P.M. (2003). Educación Social y medios de comunicación. En J. García Molina (coord.). De nuevo, la Educación Social (pp. 139-156). Madrid: Dykinson.

Petrus, A. (2000). Nuevos ámbitos en educación social. En M. Románs, A. Petrus y J. Trilla. De profesión educador(a) social (pp. 63-147). Barcelona: Paidós.

Resolución de 16 de octubre de 2006, de la Dirección General de Gestión de Recursos Humanos, por la que se realiza convocatoria para la cobertura provisional durante el curso 2006-2007 en puestos de educación social en el ámbito educativo. Boletín Oficial de la Junta de Andalucía, 213 de 3 de noviembre de 2006.

Resolución de 26 de septiembre de 2017, por la que se ordena la publicación del Convenio de Colaboración entre la Consejería de Educación y Universidades y el Colegio Profesional de Educadores y Educadoras Sociales de Canarias (CEESCAN) para la mejora del sistema educativo y de la educación social, Botetín Oficial de Canarias, $n^{\circ} 192$ de 4 de octubre de 2017.

Senent, J.M. (2003). Desarrollo contemporáneo de la Educación Social en Europa. Perspectiva comparada. En C. Ruiz (coord.). Educación Social. Viejos usos y nuevos retos (pp. 59-90). Valencia: Universidad de Valencia.

Sierra, J.E.; Vila, E.S.; Caparrós, E. y Martín, V.M. (2017). Rol y funciones de los educadores y las educadoras sociales en los centros educativos andaluces. Análisis y reflexiones. Revista Complutense de Educación, 28 (2), 479-495.

Trilla, J. (1996). «L'Aire de Família» de la pedagogía social. Temps d’Educació, 15, 39-57.

Vega, A. (2013). La educación social en la escuela: complemento imprescindible. RES. Revista de Educación Social. 16. http://www.eduso.net/res/pdf/16/escomple_res_16.pdf 